\title{
Utility of exome sequencing in routine care for metastatic colorectal cancer
}

\author{
MELCHIOR DE GIRAUD D'AGAY ${ }^{1,2}$, LOÏCK GALLAND ${ }^{1-3}$, ZOE THARIN $^{3}$, \\ CAROLINE TRUNTZER ${ }^{1,3,4^{*}}$ and FRANCOIS GHIRINGHELLI ${ }^{1-5^{*}}$
}

\author{
${ }^{1}$ Platform of Transfer in Biological Oncology, Georges François Leclerc Cancer Center-UNICANCER; \\ ${ }^{2}$ University of Burgundy-Franche Comté; ${ }^{3}$ Department of Medical Oncology, \\ Georges François Leclerc Cancer Center-UNICANCER; ${ }^{4}$ Genomic and Immunotherapy Medical Institute, \\ Dijon University Hospital; ${ }^{5}$ Mixed Research Unity (UMR - Unité de Recherche Mixte) 1231 - INSERM, 21000 Dijon, France
}

Received January 4, 2021; Accepted July 1, 2021

DOI: $10.3892 / \mathrm{mco} .2021 .2392$

\begin{abstract}
Metastatic colorectal cancer (mCRC) is a heterogenous disease and its prognosis depends on clinical features, such as tumor sidedness, and whether it is metachronous or synchronous. However, little is known about the overall genomic characterization of $\mathrm{mCRC}$ in these clinical subtypes. This single-center observational study included 77 patients with mCRC who underwent somatic and germline exome analysis during the first or second line of therapy in 2018. Somatic and germline variants were determined in addition to tumor mutational burden, ploidy, clonality, human leucocyte antigen typing, neoantigens, and mutational and copy number signatures. Variables associated with sidedness, synchronous status and RAS status were determined using Fisher's test; and variables associated with overall survival were determined using univariate Cox survival models. The present study successfully generated whole exome sequencing analysis in $77 \mathrm{mCRC}$ cases. Among them, 50 were left- and rectal-sided, while 27 were right-sided. Furthermore, 27 were metachronous and 46 were RAS-mutated. The median OS was 3.75 years. It was observed that signature single nucleotide variation (SNV) 26, oncogenic alterations in receptor tyrosine kinase and nucleotide excision repair pathways were associated with tumor sidedness. SNV signature 3, Hedgehog signaling and mismatch repair pathways were associated with synchronous status. Phosphatidylinositol signaling system, ERK signaling and chromatin organization pathways were associated with RAS mutant status. In the whole cohort, metachronous
\end{abstract}

Correspondence to: Professor Francois Ghiringhelli, Department of Medical Oncology, Georges François Leclerc Cancer Center-UNICANCER, 1 Rue du Professeur Marion, 21000 Dijon, France

E-mail: fghiringhelli@cgfl.fr

${ }^{*}$ Contributed equally

Key words: colorectal cancer, exome, biomarkers, sidedness, metachronous, synchronous, RAS status, genomic characterisation metastasis was associated with improved survival. On gene variation, PTEN, PDGFRA, MYCN and SMAD4 were associated with poor prognosis, as was SNV signature 15. In conclusion, this study highlighted that structural and pathway genomic features are associated with sidedness, synchronous status, RAS status and overall survival and could be helpful to improve the stratification of patients with colorectal cancer.

\section{Introduction}

Colorectal cancer (CRC) is the second leading cause of cancer death worldwide (1). The estimated number of deaths due to CRC in Europe was 243,000 in 2018 (2). Approximately $25 \%$ of patients present metastases at the time of diagnosis (synchronous disease) and about half of the remaining patients will develop metachronous metastases, contributing to the high mortality rates reported for CRC (3).

The treatment of metastatic CRC (mCRC) is based on chemotherapy when metastases cannot be removed by surgery. Treatment is guided by molecular information. Microsatellite unstable tumors are treated with immunotherapy (4), while microsatellite stable tumors, which account for $95 \%$ of $\mathrm{mCRC}$, are treated by cytotoxic agents and targeted therapies. The principal cytotoxic agents currently used are fluoropyrimidines, irinotecan and oxaliplatin. The chemotherapy regimen often consists of fluorouracil and folinic acid combined with either oxaliplatin (FOLFOX) or irinotecan (FOLFIRI) or both (FOLFOXIRI). These chemotherapies are associated with two different classes of target therapies: Anti-angiogenesis drugs, and anti-epidermal growth factor receptor (EGFR). While antiangiogenics can be used broadly $(5,6)$, anti EGFR therapy is only effective in patients with RAS wild type status $(3,7)$.

It is currently established that $\mathrm{mCRC}$ is a heterogeneous disease. Tumor sidedness carries strong prognostic value (8) while synchronous $\mathrm{mCRC}$ is associated with a particularly poor prognosis. These data suggest that the biological characteristics of these tumors are different. Several papers have introduced CRC molecular subtyping systems, which are currently summarized in consensus molecular subtypes (9). This transcriptomic typology classifies patients into 4 consensual subtypes with different prognostic behavior. 
Using large panel sequencing data, a recent analysis detailed the molecular landscape of mCRC (10). This study observed different gene variations between left and right-sided tumors. However, the genomic structural pattern was not assessed in this study.

Here, using exome analysis of $77 \mathrm{mCRC}$ patients, we aimed to determine genomic structural patterns and gene or pathway variations associated with overall survival, sidedness, RAS mutation status and synchronous disease.

\section{Materials and methods}

Study population. Seventy-seven patients with mCRC in whom WES (Whole Exome Sequencing) analysis was performed in 2018 as part of routine care, and interpreted according to the Molecular Tumor Board of the Georges François Leclerc Cancer Center, were included in this single-centre, retrospective study. WES analyses were performed during first or second line therapy. WES analysis is performed as part of routine care in our center in order to find potential targetable mutations for second line therapy. Before patients consented to WES of their tumoral tissue, they were informed by their oncologist. Germline testing was performed after counseling by a clinical geneticist.

Only patients from whom informed consent was obtained and recorded in the medical chart were included in this retrospective study. The study was approved by the CNIL (French national commission for data privacy) and the local ethics committee, and was performed in accordance with the Helsinki Declaration and European legislation.

Sample selection. Physicians selected an archival tumor sample (primary or metastasis) for genomic analysis. At the discretion of the physician, a new tumor biopsy could be proposed to the patient. Tumor cellularity was assessed by a senior pathologist on a hematoxylin and eosin slide from the same biopsy core used for nucleic acid extraction and molecular analysis.

DNA isolation. DNA was isolated from archival tumor tissue using the Maxwell 16 FFPE Plus LEV DNA purification kit (Promega Corp.). DNA from whole blood (germline DNA) was isolated using the Maxwell 16 Blood DNA Purification kit (Promega Corp.) following the manufacturer's instructions. The quantity of extracted genomic DNA was assessed by a fluorometric method with a Qubit device.

Whole exome capture and sequencing. Two hundred ng of genomic DNA were used for library preparation, using the Agilent SureSelectXT reagent kit (Catalog number G9642B, Agilent Technologies, Inc.)and the All Exon v5 probeset (5190-8863, Agilent Technologies, Inc.). Following hybridization, the libraries were purified according to the manufacturer's recommendations and amplified by polymerase chain reaction (12 cycles). DNA integrity was verified using TapeStation (SCREENTAPE D1000 tapestation 5067-5582, reagents D1000 tapestation 5067-5583). Concentrations were measured using Qubit ${ }^{\circledR}$ dsDNA BR Assay Q32853. Loading concentrations were $22 \mathrm{nM}$ for fragmentation and $6 \mathrm{pM}$ for NextSeq injection. Normalized libraries were pooled, and DNA was sequenced on an Illumina NextSeq500 device using 2x111-bp paired-end reads and multiplexed. Names, catalog numbers and suppliers of the Illumina sequencing kit were following: NextSeq 500 High Output Kit FC404-2004/2140817 and NextSeq 500 Mid Output Kit FC404-2003/2140816. More than $90 \%$ of the target sequence was covered with a read depth of at least $10 \mathrm{X}$ for somatic DNA.

Exome analysis pipeline. Reads in FASTQ format were aligned to the reference human genome GRCh37 using the Burrows-Wheeler aligner (BWA v.0.7.15). Local realignment was performed using the Genome Analysis Toolkit (GATK v.3.6). Duplicate reads were removed using Picard v.2.5. To identify somatic single-nucleotide variants (SNVs), a validated pipeline was used that integrates mutation calls from three different mutation callers. Single Nucleotide Variants (SNVs) were called with VarScan (v2.4.3) (11) and Mutect (v1.1.7) (12) insertion/deletions (indels) were called with VarScan and Strelka (v2.9.2) (13). Tumor Mutational Burden (TMB) was calculated using the number of significant SNV (UTRs, synonyms, introns and intergenic SNVs filtered out) divided by the number of megabases covered at a defined level. TMB was calculated with and without splicing sites mutations. Splicing site mutations were excluded because it has been demonstrated that variants present in splice regions have predominantly no impact (14). To identify tumor-specific mutant peptides, pVAC-Seq v4.0.3 (15) (personalized Variant Antigens by Cancer Sequencing) was used; pVAC-Seq is based on HLA typing obtained by HLAminer (16). TITAN (17) was used to infer the number of copy number alterations (CNA) subclones, the number of large deletions, as well as loss of heterogeneity $(\mathrm{LOH})>15 \mathrm{Mb}$ from whole-exome sequencing data. It was also used to estimate tumor ploidy. SNV signatures were generated using DeconstructSigs (v1.8.0) (18) and COSMIC signatures identified by Alexandrov et al (19). CNV signatures were inferred according to the methodology of Macintyre et al (20). MSI score was computed using MSIsensor (21) HRD score was obtained through scarHRD (22) pipeline.

Statistical analysis. Patient and disease characteristics were compared across the different groups of interest using the Chi-2 or Fisher's exact test for qualitative variables and the Wilcoxon test for continuous variables, as appropriate. Enrichr analysis using KEGG database was performed on genes differentially mutated given sidedness, metastases and KRAS mutation status (23). Genes with a P-value $<0.1$ were selected for this analysis. Enrichr is a web-based tool for analysing gene sets; it returns any enrichment of common annotated biological features, here KEGG database.

Survival analysis was performed using the survival $\mathrm{R}$ library. Continuous variables were dichotomised using Lausen et al (24) methodology through the maxstat library (25). The prognostic value of the different variables was tested using univariate Cox regression for overall (OS) survival. OS was defined as the time from diagnosis to death from mCRC. Survivors were censored at the end of study. Survival probabilities were estimated using the Kaplan-Meier method and survival curves were compared using the log-rank test. 
Table I. Description of treatment based on molecular tumour board recommendation.

\begin{tabular}{|c|c|c|c|c|c|}
\hline Sample & Somatic mutation & Nucleotide variant & Protein variant & Impact & Treatment \\
\hline 1 & BARD1, BRIP1 & $\begin{array}{l}\text { c. } 266 \mathrm{C}>\mathrm{T}, \\
\text { c. } 2665 \mathrm{C}>\mathrm{A}\end{array}$ & $\begin{array}{l}\text { p.Pro89Leu, } \\
\text { p.Gln889Lys }\end{array}$ & Unknown & OLAPARIB \\
\hline 2 & NF1 & c. $1007 \mathrm{G}>\mathrm{A}$ & p.Trp336Ter & Unknown & TRAMETINIB \\
\hline 3 & BRAF & c. $1799 \mathrm{~T}>\mathrm{A}$ & p.Val600Glu & Activating function & VEMURAFENIB \\
\hline 4 & RAD51C & c. $859 \mathrm{~A}>\mathrm{G}$ & p.Thr287Ala & Loss of function & OLAPARIB \\
\hline 5 & MSH6 & c. $2017 \mathrm{C}>\mathrm{A}$ & p.Pro673Thr & Unknown & NIVOLUMAB \\
\hline 6 & TOP1 & c. $852 \mathrm{G}>\mathrm{A}$ & p.Lys284Lys & Unknown & IRINOTECAN \\
\hline 7 & TP53 & c.450_451delAC & p.Pro152AlafsTer28 & Loss of function & AFLIBERCEPT \\
\hline 8 & KRAS & c. $35 \mathrm{G}>\mathrm{C}$ & p.Gly 12Ala & Activating function & TRAMETINIB \\
\hline 9 & MSH6 & .3254 delC & p.Phe1088SerfsTer2 & Loss of function & DURVAUMAB \\
\hline 10 & TP53 & c. $638 \mathrm{G}>\mathrm{T}$ & p.Arg213Leu & Loss of function & BEVACIZUMAB \\
\hline 11 & MSH6 & c. $2731 \mathrm{C}>\mathrm{T}$ & p.Arg911Ter & Unknown & NIVOLUMAB \\
\hline 12 & $\begin{array}{l}\text { High number } \\
\text { of variant }\end{array}$ & - & - & - & NIVOLUMAB \\
\hline 13 & KRAS & c. $35 \mathrm{G}>\mathrm{T}$ & p.Gly 12Val & Activating function & TRAMETINIB \\
\hline 14 & MTOR & c. $6352 \mathrm{C}>\mathrm{T}$ & p.Leu2118Phe & Unknown & EVEROLIMUS \\
\hline 15 & TP53 & c. $637 \mathrm{C}>\mathrm{T}$ & p.Arg213X & Loss of function & REGORAFENIB \\
\hline 16 & KRAS & c. $38 \mathrm{G}>\mathrm{A}$ & p.Gly13Asp & Activating function & TRAMETINIB \\
\hline
\end{tabular}

Statistical analyses were performed using the $\mathrm{R}$ software (http://www.R-project.org/) and graphs were drawn using GraphPad Prism version 7.03 (GraphPad Software, LLC).

\section{Results}

Patients' clinical characteristics. We included 27 (35\%) patients with right primary colon cancer and $50(65 \%)$ with rectal or left primary colon cancer. Twenty-seven $(35 \%)$ patients had metachronous metastasis and $50(65 \%)$ were synchronous. Forty-six (60\%) patients were RAS mutated. The most frequent metastasis site was the liver (54 patients, 70\%). Liver metastasis were more frequently synchronous (44 patients, 57\%), than metachronous (10 patients, 12\%) $(\mathrm{P}$-value=0.01). The most common sites of first metastasis were the liver (74\%) and the lung (27\%), two metastatic sites that are potentially curable by resection. The other identified sites of metastases were the lymph nodes, peritoneal, adrenal and bone metastasis.

The presence of lung or liver metastases at time of diagnosis of metastatic disease did not vary significantly by primary tumour site (19\% of right-sided tumors versus $32 \%$ of left-sided mCRC for lung metastasis, and $18 \%$ of right-sided tumors versus $36 \%$ of left-sided mCRC for liver metastasis). In contrast, peritoneal and omental metastases were more frequent among right-sided primary tumors $(\mathrm{P}$-value $=0.001)$. The main differences between clinical variables according to tumor sidedness are presented in Table SI, according to synchronous or metachronous status in Table SII, and between RAS/WT mutations in Table SIII.

All patients received a doublet or triplet of chemotherapy as first-line therapy; fluorouracil and folinic acid were used consistently. Patients with synchronous metastasis significantly more frequently received oxaliplatin in their chemotherapy regimens $(\mathrm{P}$-value $=0.01)$. Thirty patients were treated with anti-epidermal growth factor receptor (EGFR), without any significant difference concerning the sidedness or the time to metastasis (P-values 0.13 and 0.61 respectively). Sixty-four patients $(83 \%)$ were treated with anti-angiogenesis drugs (bevacizumab or aflibercept). Fifty-four patients (70\%) received a therapeutic proposition from the molecular tumor board. Only 18 patients $(23 \%)$ received a treatment based on the molecular tumour board recommendations, most frequently using an oral MEK-inhibitor called Trametinib (Table I). Three patients yielded significant clinical benefit from these therapeutic strategies, with more than 6 months of progression-free survival. Two of these three patients had unstable microsatellite status and were treated with immunotherapy.

Patients' genomic characteristics. The most frequent mutation in the whole cohort was APC followed by TP53 and RAS (Fig. 1A). A summary of the genomic characteristics is presented in Table II. Thirty one patients presented WT mutational status, 44 patients presented KRAS and 2 patients NRAS mutational status. We identified 8 patients $(10 \%)$ with BRAF mutation. The most frequent RAS mutation was KRAS (60\%).

Mean tumor mutational burden (TMB) without splicing regions in the whole cohort was 8.87 (median $=5.50, \mathrm{IQR}=3.07$ ). The mean number of neoantigens was 13 (median $=11$, $\mathrm{IQR}=7.75$ ). Five patients had a high HRD score, using the classical cut off value 42 (26). The two most frequent SNV signatures were 1 and 25 . The two most frequent CNV signatures were 3 and 5 .

Seventy-two (94\%) patients had proficient mismatch repair status (pMMR) and $5(6 \%)$ had deficient mismatch repair status (dMMR) on immunohistological analysis (Table SIV). Median MSI score and TMB score were significantly higher in the $\mathrm{dMMR}$ group (respectively $\mathrm{P}$-value $=0.02$ and $\mathrm{P}$-value $=0.01$ ) 
A

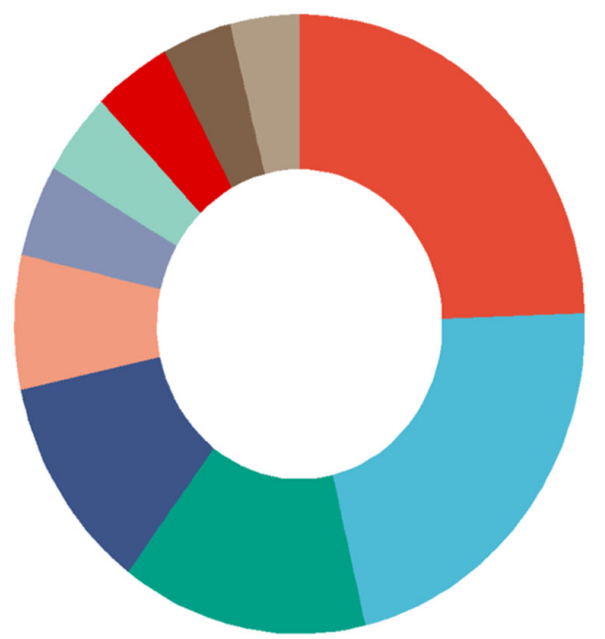

B
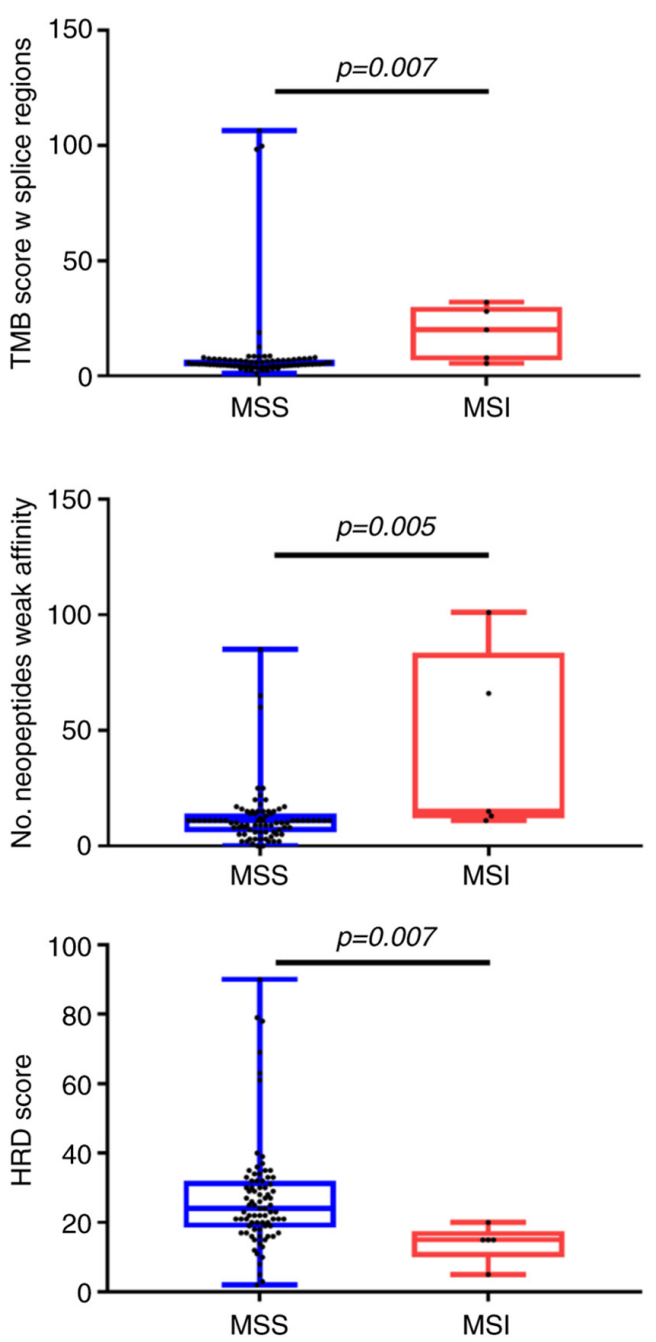

Genes
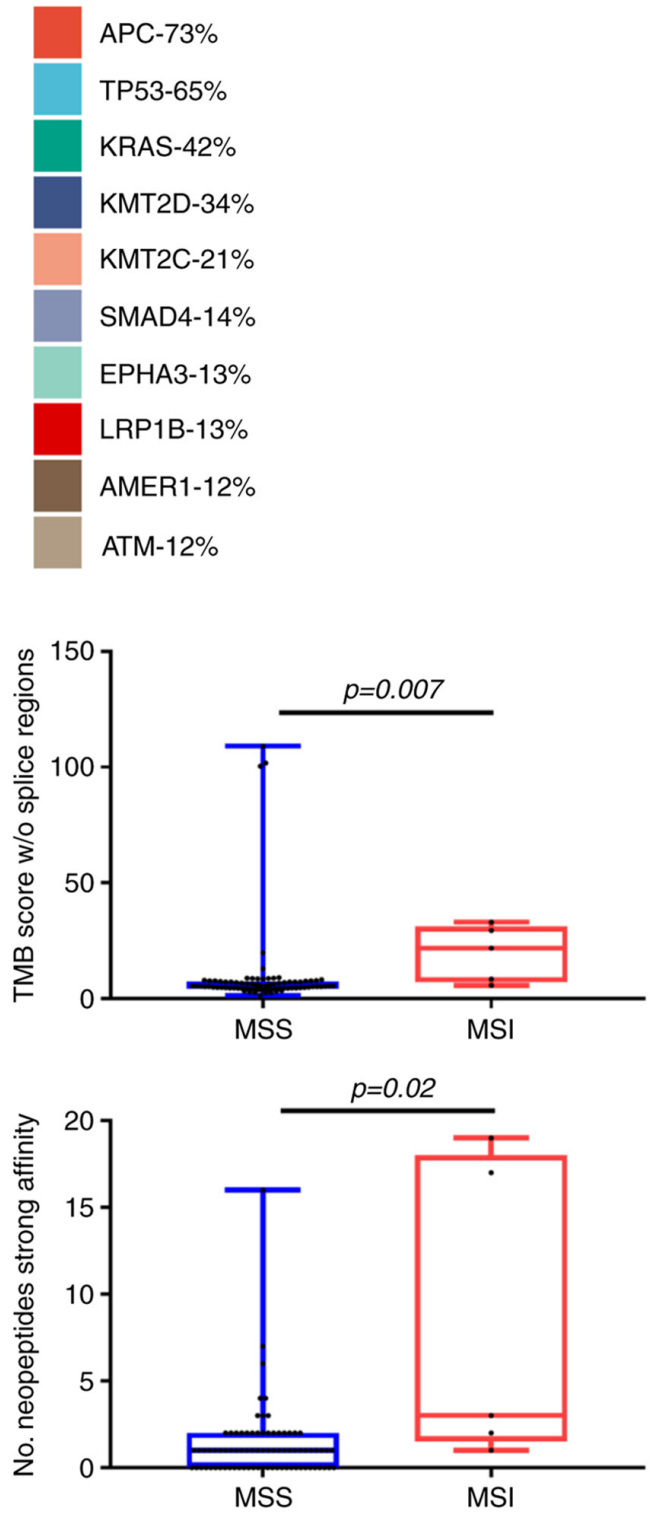

Figure 1. Genetic characteristics in the whole cohort. (A) Donut representing the proportion of patients presenting each of the top 10 mutated genes. (B) Boxplots representing the distribution of genomic characteristics that were significantly different between MSS and MSI patients, namely TMB score with and without splice regions, number of neopeptides according to whether they present weak or strong affinity, and HRD score. P-values were obtained using Wilcoxon tests. TMB, tumor mutational burden; MSS, microsatellite stability; MSI, microsatellite instability; HRD, homologous recombination deficiency.

(Fig. 1B). There was no significant enrichment of signature 6 in patients with dMMR status (P-value $=0.7)$.

Association of genomic variables with sidedness. To evaluate whether we could isolate a genetic basis for the difference in survival between disease that originates in the right versus left side of the colon, we analysed genomic structural and gene alterations for the primary tumor site. The only difference in structural variants was significant enrichment of signature 26 in left-sided primary tumors (Fig. 2A). Signature 26 is 


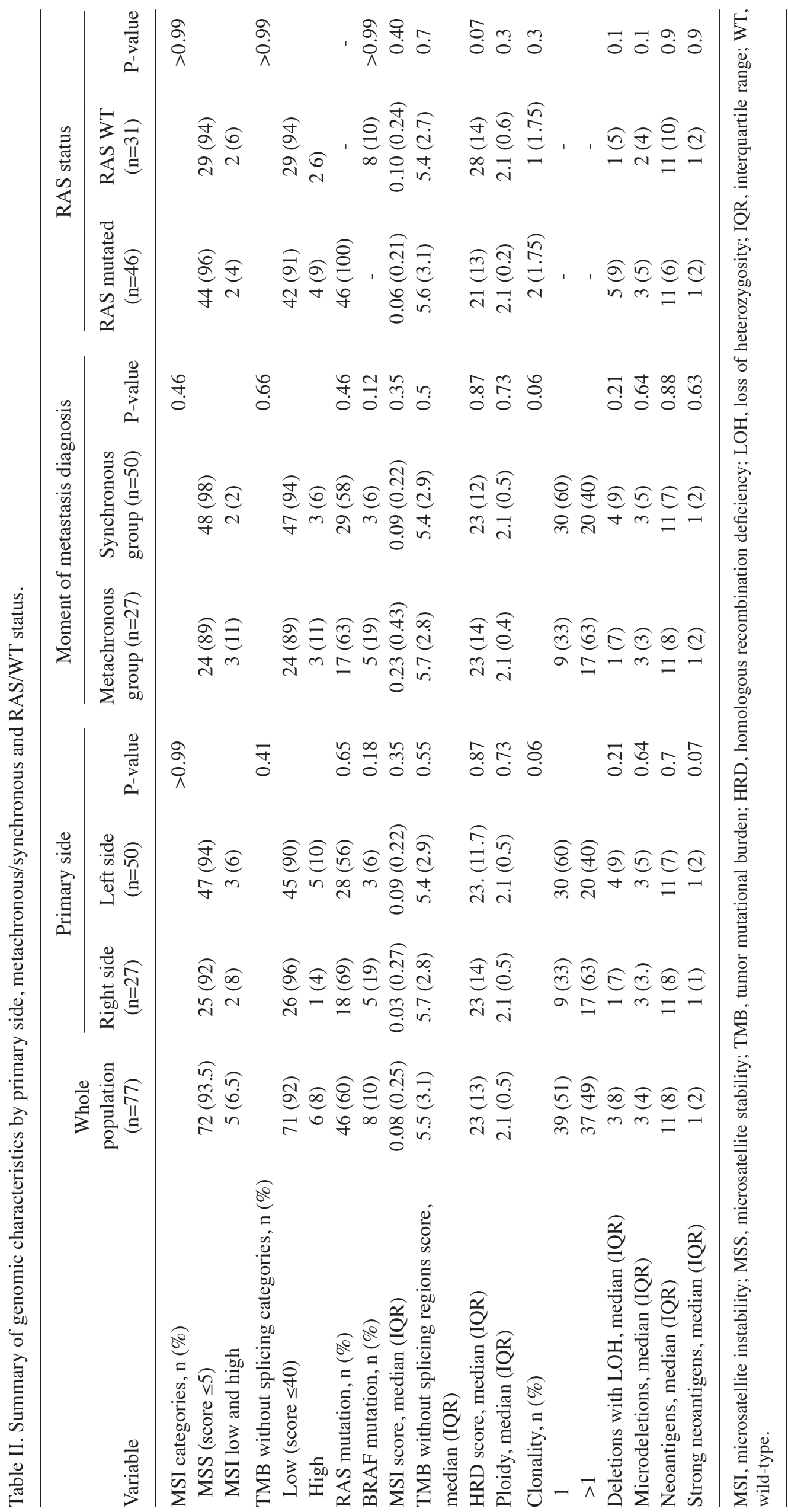


A

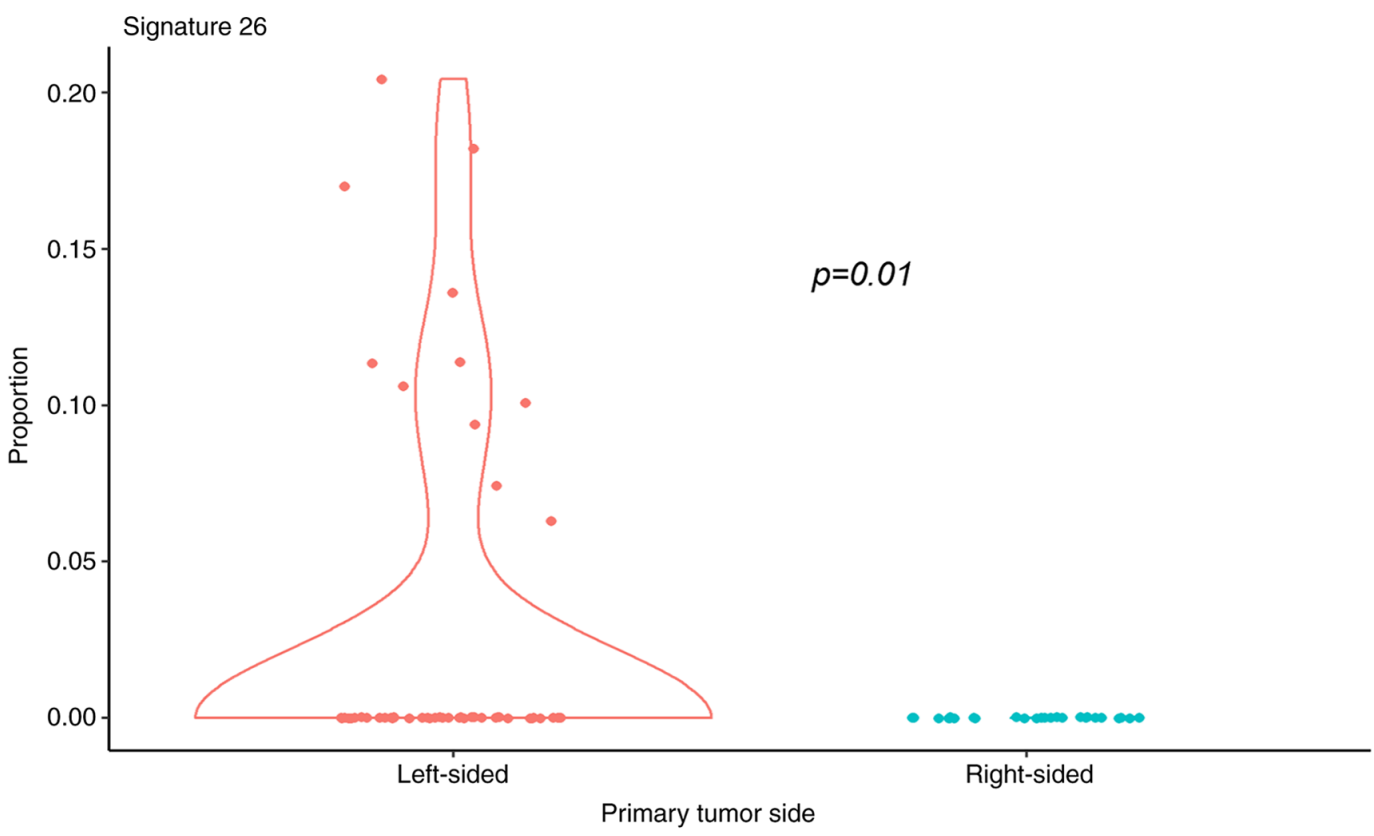

B

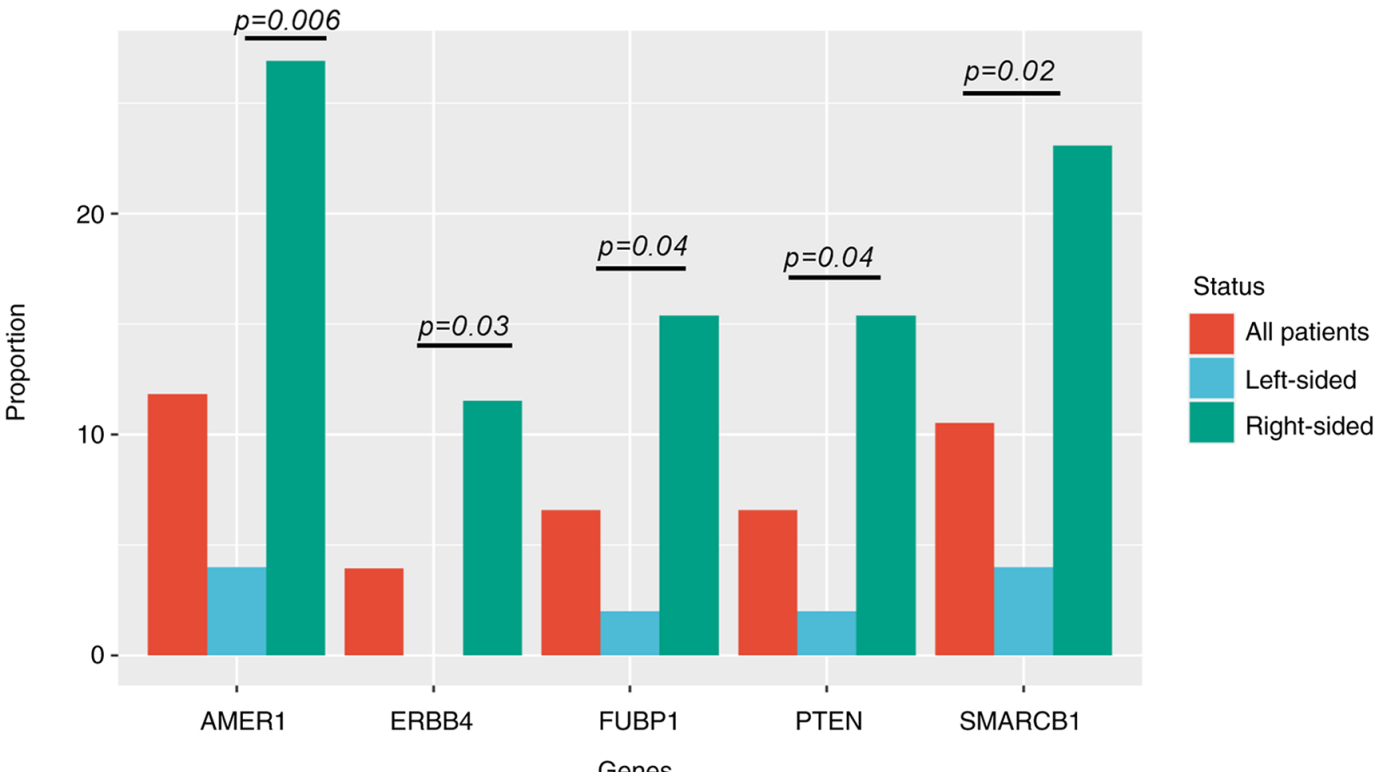

C
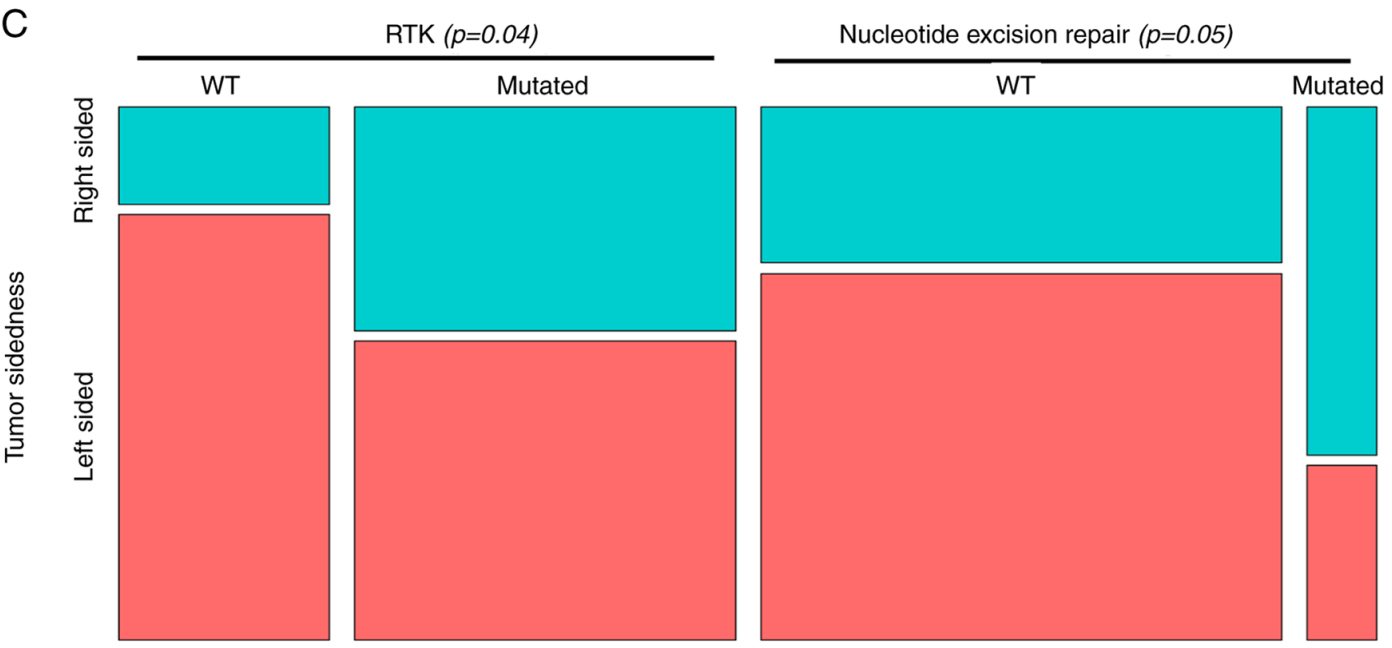

Pathway

Figure 2. Genetic characteristics discriminating tumor sidedness. (A) Distribution of the proportion of single nucleotide variation signature 26 according to primary tumor side. (B) Proportion of patients presenting at least one mutation in genes for which Fisher tests were considered significant $(\mathrm{P}<0.05)$, between primary tumor sides, by group and in the whole cohort. (C) Distribution of patients presenting an alteration in significant signaling pathways by primary tumor side. Only pathways for which the Fisher tests were considered significant $(\mathrm{P}<0.05)$ are presented. RTK, receptor tyrosine kinase; WT, wild-type. 
associated with homologous repair deficiency (defective DNA mismatch repair).

For gene base analysis (Fig. 2B), there was significant enrichment of oncogenic alterations in AMER1, SMARCB1, ERBB4, FUBP1 and PTEN in right-sided primary tumors. Nevertheless, we did not identify any significant differences according to sidedness or frequencies of KRAS/BRAF mutations. Mutations observed in these genes are further described in Table SV. Results of Enrichr analysis using KEGG database are described in Table SVI.

Beyond the gene-level associations, analysis at the level of oncogenic pathways showed that mutations related to certain pathways differed according to primary tumor site. These pathways consisted in significant enrichment of Nucleotide excision repair for right-sided tumors, and RTK for left-sided ones (Fig. 2C). No significant association was found between sidedness and TP53/ATM, WNT/CTNNB1, TGF-beta or IGF2/PI-3-kinase pathways, which are frequently mutated in CRC.

Association of genomic variables with synchronous versus metachronous presentation. Using structural genome analysis, SNV signature 3, which is associated with failure of DNA double-strand break repair by homologous recombination, was significantly associated with synchronous presentation (Fig. 3A). We did not find additional differences at this genomic level.

P53 loss of function mutations were significantly more frequent in synchronous metastasis. In contrast, metachronous metastasis was associated with PTCH, PTPRD and CSF1R mutations (Fig. 3B). Mutations observed in these genes are further described in Table SV. Results of Enrichr analysis using KEGG database are described in Table SVI.

When pooling mutations in gene pathways, we observed that Mismatch repair and Hedgehog signaling were significantly more frequently affected in metachronous tumors (Fig. 3C).

Association of genomic variables with RAS status. In our series, the prevalence of RAS mutations was not significantly different according to tumor location. On gene variation, KMT2B and RET were significantly associated with RAS wild type tumors. In contrast, KMT2C and GNAS were significantly more frequent with RAS mutated tumors (Fig. S1A). Mutations observed in these genes are further described in Table SV. Results of Enrichr analysis using KEGG database are described in Table SVI.

An analysis at the level of oncogenic pathways demonstrated that ERK signaling and chromatin organisation pathways were significantly associated with RAS mutation, while Phosphatidylinositol signaling was associated with WT tumors (Fig. S1B).

Association of genomic variables with survival. Median OS was 3.75 years. Regarding genomic structural variants, low SNV signature 15 was associated with poor overall survival (Fig. 4A). In gene variation, PDGFRA, SMAD4, PTEN, MYCN mutations were significantly associated with poor prognosis (Fig. S2A-D). We also estimated a multivariate model involving the 4 genes; all genes remain significant. At the level of oncogenic pathways, cell-cycle, NFKappaB signaling and mismatch repair pathways were significantly associated with better survival (Fig. 4B-D). We did not observe a significant association between any WNT-signaling pathway and CRC survival.

Overall survival was significantly better in patients with metachronous metastatic disease, but this difference was no longer significant after removing MMR deficient tumors, thus suggesting a link between the prognosis of metachronous disease and enrichment in MMR deficient tumors (Fig. 4E and F).

\section{Discussion}

CRC is caused by multiple risk factors, including environmental, lifestyle and genetic risks. All these elements cause mutations and epigenetic alterations, conferring on cells the capacity to transform and grow, with aberrant DNA editing and defective DNA maintenance.

Next-generation sequencing (NGS) has identified a diversity of driver mutations in genes and altered signaling pathways in CRC $(10,27)$. In addition, genomic structural patterns within gene pathway mutations could be used to identify prognostic features. Recent studies have revealed a mutational landscape of colorectal cancer and defined different subtypes that could guide therapeutic decisions (28). Increasing access to WES and the increasing rapidity of analysis offers new opportunities to implement such tests in the care of mCRC. WES analysis is performed in routine care in our center in order to find potentially targetable mutations for second line therapy.

Like in previous studies of precision medicine, we found that only very few $\mathrm{mCRC}$ patients yielded a benefit from precision medicine. In our study, we used WES analysis to identify genomic mutational profiles linked to tumor sidedness and metastatic occurrences, as well as genomic features related to prognosis.

In a systemic review and metanalysis (8), left-sided primary tumors were associated with improved prognosis, for both localized and metastatic tumors, in comparison to right-sided disease. Peritoneal and omental metastases, which are metastatic sites known to be associated with poor survival (29), were more frequent among right-sided primary tumors. Moreover, the survival differences seen between patients with right-sided vs. left-sided primary tumor sites in mCRC are supported by differences in transcriptomic patterns $(8,30)$.

Like other authors $(30,31)$, we showed that metachronous metastatic status is associated with better outcome. However, the molecular mechanism remains obscure, and the prognostic role seems to be less impactful than that of MMR status.

Our findings indicate that differences in survival could also be explained by genomic differences. Our analysis relied on structural information and on mutated genes grouped in pathways. We showed in particular that SNV signature 26, associated with MMR deficiency 29 (https://cancer.sanger. ac.uk/cosmic/signatures_v2.tt), was surprisingly associated with left-sided mCRC, while SNV signature 3 was associated with synchronous metastasis. Signature 3 is associated with better overall survival in ovarian (32) and breast cancer (33), by the better response to platinum therapy. At present, no data link signature 3 to oxaliplatin efficacy in mCRC. This question may be relevant for further clinical trials. 
A

A Signature 3

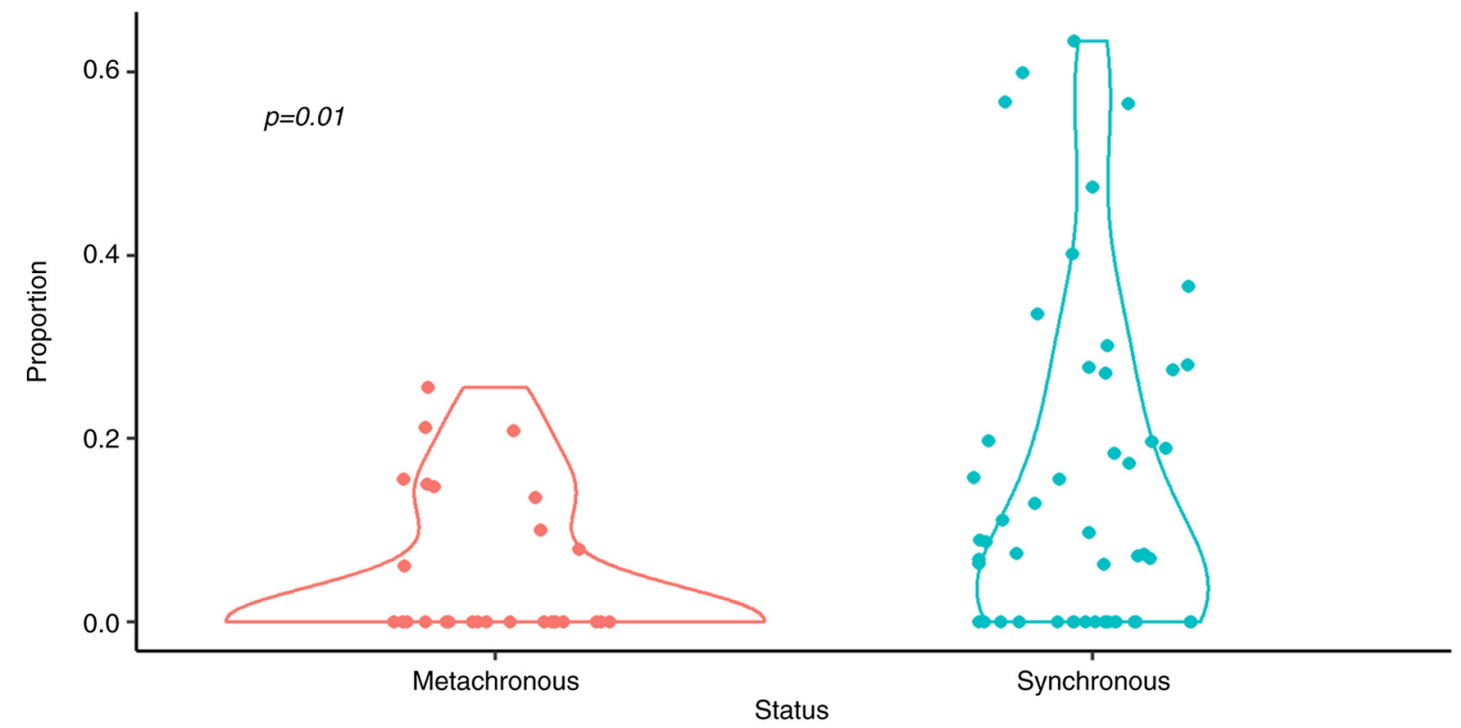

B

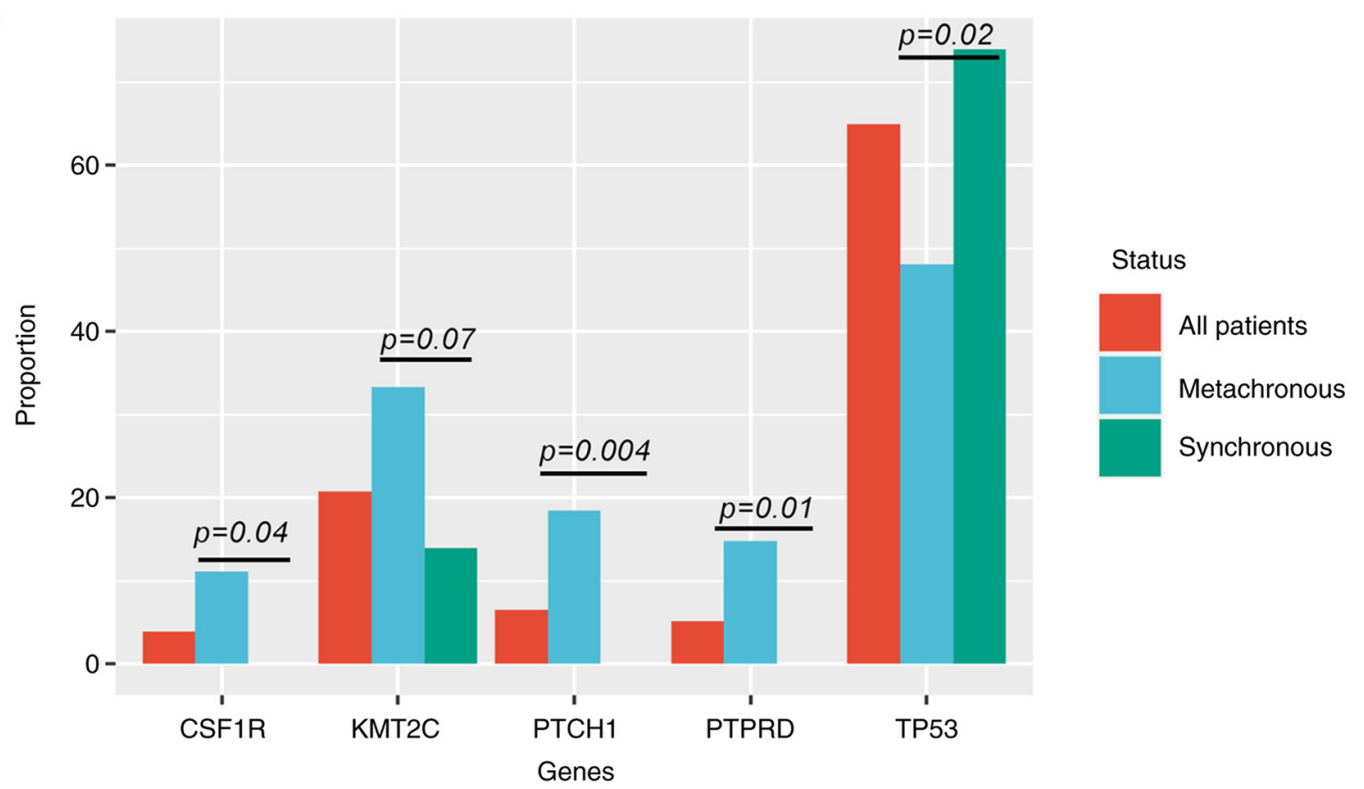

C

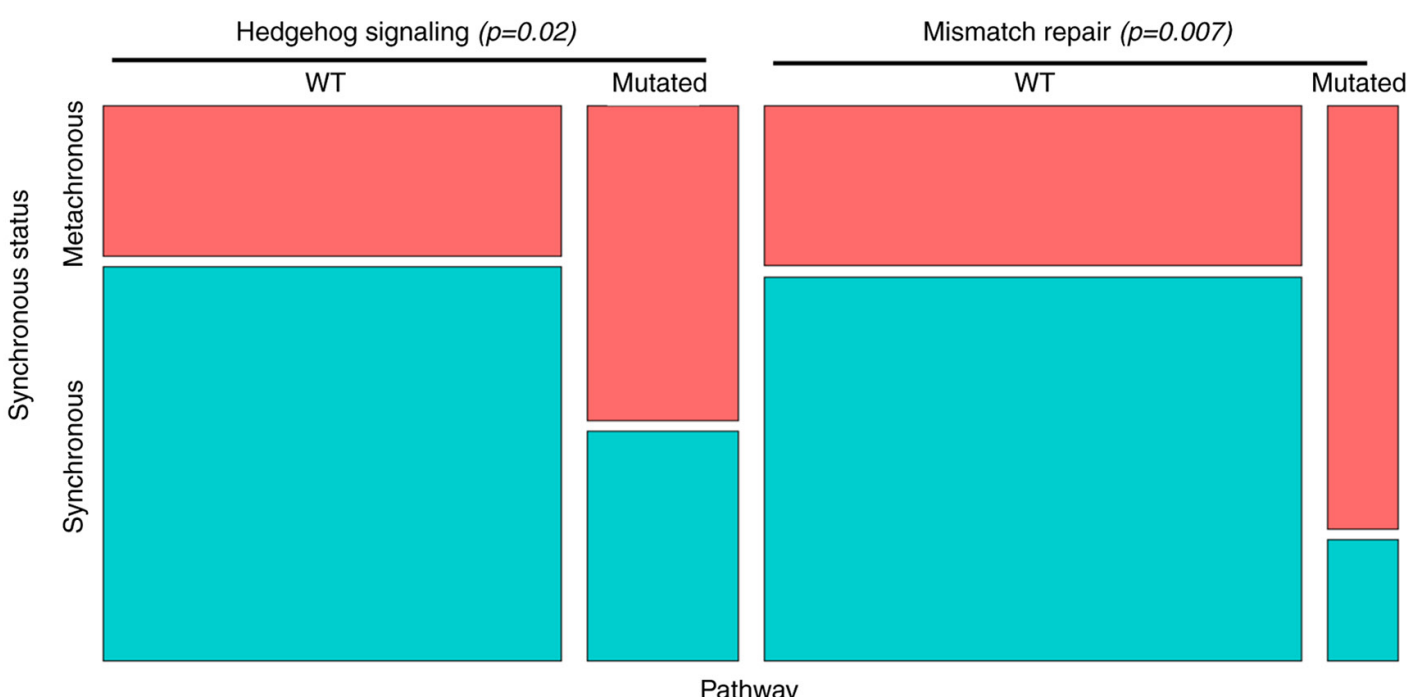

Figure 3. Genetic characteristics discriminating synchronous or metachronous disease. (A) Distribution of the proportion of SNV signature 3 according to synchronous or metachronous status. (B) Proportion of patients presenting at least one mutation in genes for which the Fisher tests were considered significant $(\mathrm{P}<0.05)$, between synchronous or metachronous status, by group and in the whole cohort. (C) Distribution of patients presenting an alteration in significant signaling pathways by synchronous or metachronous status. Only pathways for which the Fisher tests were considered significant $(\mathrm{P}<0.05)$ are presented. WT, wild-type. 

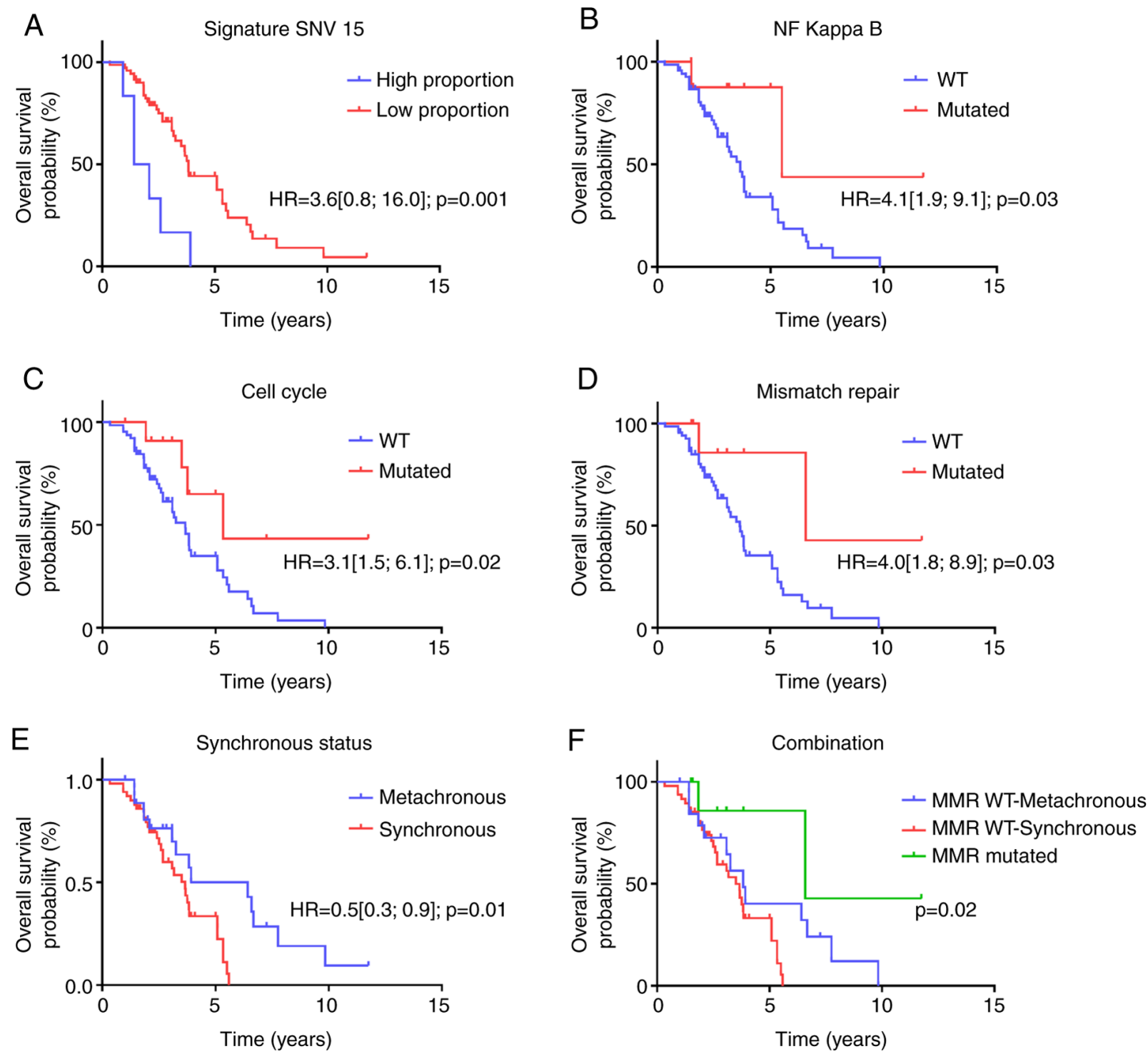

Figure 4. Genomic variables and overall survival. Kaplan Meier curves for variables with significantly different overall survival according to: (A) SNV signature 15, (B) oncogenic pathways NF Kappa B, (C) cell cycle, (D) mismatch repair, (E) synchronous status and (F) combination of mismatch repair and synchronous status. HR, hazard ratio; MMR, mismatch repair; SNV, single nucleotide variation; WT, wild-type.

Moreover, somatic mutations in TP53 genes, which are present in the majority of cancers, are associated with poorer clinical outcomes, in several cancer types, including CRC (28). P53 loss of function mutations were significantly more frequent in synchronous metastasis.

In contrast, when we looked at more classical genomic features such as tumor mutational burden, there was no significant difference between tumors according to sidedness or time to metastasis occurrence. Conversely, we observed that MMR deficiency was associated with better outcome. MMR deficiency is more frequent in metachronous tumors and this genetic event may explain the better prognosis of these patients.

Oncogenic alterations-KRAS, NRAS and BRAF, which exhibit resistance to EGFR therapy with panitumumab and cetuximab, did not differ in our cohort, as previously reported (34), according to sidedness or metastatic occurrence, thus suggesting that these parameters are probably weak prognostic factors. The RAS pathway is a cell signaling pathway that plays a key role in regulation of cellular proliferation, apoptosis, cellular differentiation and migration and angiogenesis. This pathway is often dysregulated in mCRC. The association between RAS mutations and activation of the ERK signaling pathway is already well known.

When we looked at classical somatic mutations in mCRC, we observed that APC was frequently mutated in our series. However, APC was not associated with survival. The WNT signaling pathway is also frequently mutated in CRC and we did not observe a significant association between any WNT-signaling pathway and CRC survival. SMAD4 loss was found to be associated with poor outcome in our series. Similarly, a recent report also showed an association between SMAD4 loss and poor CRC survival, resistance to chemotherapy and decreased tumor immune infiltration (35).

Both PDGF and PDGFR families play an important role in colorectal carcinogenesis, and PDGFR is frequently overexpressed in CRC. Activation of this pathway is frequently related 
to angiogenesis, invasion, metastasis and poor survival (36). In our study, mutations in PDGFRA were associated with poor prognosis.

The main limitation of our work is the low number of patients included, which may impact the statistical significance, and precluded multivariate analysis. Moreover, WES was made during first or second line of treatment, so mutation profiles were generated under therapeutic pressure, which changed the cancer characterizations. Unfortunately, we do not dispose of samples before treatments as a baseline. In addition, considering the sequencing result of PTEN, PDGFRA, MYCN or SMAD4, the lack of PCR verification step is a limitation of this study and should be done in future works. Thus, our results should be considered as descriptive and exploratory, and warrant confirmation in further studies including larger sample sizes.

In conclusion, with the development of targeted therapies, it seems necessary to be able to rapidly identify the molecular status of mCRC tumors. Large panel or exome sequencing is slightly effective in improving patient care with precision medicine; however, such analyses could reveal structural and pathway genomic features that are associated with sidedness, synchronous status, and overall survival. Although the cost of NGS is steadily declining, WES nonetheless remains expensive, and currently, this is one of the major limitations on the routine use of exome sequencing. Moreover, the number of tumour mutations that could be used to treat a given patient is limited in conventional clinical practice or clinical trials, and it is difficult to determine which patients will clinically benefit from exome sequencing. In all likelihood, before being used in routine practice, exome sequencing will be reserved for patients with advanced mCRC, after one or more lines of treatment, or for patients with very poor outcomes, who fail to respond to classical targeted therapy and chemotherapy. Indeed, the knowledge of the molecular status could lead to inclusion in therapeutic clinical trials with a direct benefit for our patients. More generally, patients with right-sided mCRC, synchronous metastasis or peritoneal and omental metastasis may benefit the most from such analysis. This important information could be used to improve patient stratification for clinical trials, and will lead to a new molecular classification of patients that could be helpful to finetune the future of mCRC therapy.

\section{Acknowledgements}

The authors would like tothankMs.Sandy Chevrier(Department of Medical Oncology, Georges François Leclerc Cancer Center-UNICANCER, Dijon, France), Dr Romain Boidot (Department of Medical Oncology, Georges François Leclerc Cancer Center-UNICANCER, Dijon, France) for providing whole exome sequencing data, Mr. Hugo Mananet (Department of Medical Oncology, Georges François Leclerc Cancer Center-UNICANCER, Dijon, France) for pre-processing data, and Dr Juliette Albuisson (Department of Medical Oncology, Georges François Leclerc Cancer Center-UNICANCER, Dijon, France) for her valuable comments. The authors would also like to thank Dr Fiona Ecarnot (EA3920, University of Franche-Comté, Besançon, France) for English correction and helpful comments.

\section{Funding}

No funding was received.

\section{Availability of data and materials}

The datasets generated and analyzed during the current study are available in the Sequence Read Archive repository, (https://www.ncbi.nlm.nih.gov/sra/?term=SRP318854).

\section{Authors' contributions}

MDGDA, LG, ZT, CT and FG contributed to the design and implementation of the research, to the analysis of the results and to the writing of the manuscript. CT and FG confirm the authenticity of all the raw data. All authors read and approved the final manuscript.

\section{Ethics approval and consent to participate}

The present study was approved by the CNIL (French national commission for data privacy) and the Georges François Leclerc Cancer Center (Dijon, France) local ethics committee (13.085), and was performed in accordance with the Helsinki Declaration and European legislation. Written informed consent was obtained from all subjects involved in the study. Only patients from whom informed consent was obtained and recorded in the medical chart were included in this retrospective study.

\section{Patient consent for publication}

Not applicable.

\section{Competing interests}

The authors declare that they have no competing interests.

\section{References}

1. Bray F, Ferlay J, Soerjomataram I, Siegel RL, Torre LA and Jemal A: Global cancer statistics 2018: GLOBOCAN estimates of incidence and mortality worldwide for 36 cancers in 185 countries. CA Cancer J Clin 68: 394-424, 2018.

2. Ferlay J, Colombet M, Soerjomataram I, Dyba T, Randi G, Bettio M, Gavin A, Visser O and Bray F: Cancer incidence and mortality patterns in Europe: Estimates for 40 countries and 25 major cancers in 2018. Eur J Cancer 103: 356-387, 2018.

3. Van Cutsem E, Cervantes A, Adam R, Sobrero A, Van Krieken JH, Aderka D, Aranda Aguilar E, Bardelli A, Benson A, Bodoky G, et al: ESMO consensus guidelines for the management of patients with metastatic colorectal cancer. Ann Oncol 27: 1386-1422, 2016.

4. Andre T, Shiu KK, Kim TW, Jensen BV, Jensen LH, Punt CJA, Smith DM, Garcia-Carbonero R, Benavides M, Gibbs P, et al: Pembrolizumab versus chemotherapy for microsatellite instability-high/mismatch repair deficient metastatic colorectal cancer: The phase 3 KEYNOTE-177 study. J Clin Oncol 38 (Suppl 18): LBA4, 2020.

5. Giantonio BJ, Catalano PJ, Meropol NJ, O'Dwyer PJ, Mitchell EP, Alberts SR, Schwartz MA and Benson AB III; Eastern Cooperative Oncology Group Study E3200: Bevacizumab in combination with oxaliplatin, fluorouracil, and leucovorin (FOLFOX4) for previously treated metastatic colorectal cancer: Results from the eastern cooperative oncology group study E3200. J Clin Oncol 25: 1539-1544, 2007. 
6. Van Cutsem E, Rivera F, Berry S, Kretzschmar A, Michael M, DiBartolomeo M, Mazier MA, Canon JL, Georgoulias V, Peeters M, et al: Safety and efficacy of first-line bevacizumab with FOLFOX, XELOX, FOLFIRI and fluoropyrimidines in metastatic colorectal cancer: The BEAT study. Ann Oncol 20: $1842-1847,2009$

7. Van Cutsem E, Lenz HJ, Köhne CH, Heinemann V, Tejpar S, Melezínek I, Beier F, Stroh C, Rougier P, van Krieken JH and Ciardiello F: Fluorouracil, leucovorin, and irinotecan plus cetuximab treatment and RAS mutations in colorectal cancer. J Clin Oncol 33: 692-700, 2015.

8. Petrelli F, Tomasello G, Borgonovo K, Ghidini M, Turati L, Dallera P, Passalacqua R, Sgroi G and Barni S: Prognostic survival associated with left-sided vs right-sided colon cancer: A systematic review and meta-analysis. JAMA Oncol 3: 211-219, 2017.

9. Guinney J, Dienstmann R, Wang X, de Reyniès A, Schlicker A, Soneson C, Marisa L, Roepman P, Nyamundanda G Angelino P, et al: The consensus molecular subtypes of colorectal cancer. Nat Med 21: 1350-1356, 2015.

10. Yaeger R, Chatila WK, Lipsyc MD, Hechtman JF, Cercek A, Sanchez-Vega F, Jayakumaran G, Middha S, Zehir A, Donoghue MTA, et al: Clinical sequencing defines the genomic landscape of metastatic colorectal cancer. Cancer Cell 33 : 125-136.e3, 2018.

11. Koboldt DC, Zhang Q, Larson DE, Shen D, McLellan MD, Lin L, Miller CA, Mardis ER, Ding L and Wilson RK: VarScan 2: Somatic mutation and copy number alteration discovery in cancer by exome sequencing. Genome Res 22: 568-576, 2012.

12. Cibulskis K, Lawrence MS, Carter SL, Sivachenko A, Jaffe D, Sougnez C, Gabriel S, Meyerson M, Lander ES and Getz G: Sensitive detection of somatic point mutations in impure and heterogeneous cancer samples. Nat Biotechnol 31: 213-219, 2013.

13. Kim S, Scheffler K, Halpern AL, Bekritsky MA, Noh E, Källberg M, Chen X, Kim Y, Beyter D, Krusche P and Saunders CT: Strelka2: Fast and accurate calling of germline and somatic variants. Nat Methods 15: 591-594, 2018.

14. Nykamp K, Anderson M, Powers M, Garcia J, Herrera B, Ho YY, Kobayashi Y, Patil N, Thusberg J, Westbrook M, et al: Sherloc: A comprehensive refinement of the ACMG-AMP variant classification criteria. Genet Med 19: 1105-1117, 2017.

15. Hundal J, Carreno BM, Petti AA, Linette GP, Griffith OL, Mardis ER and Griffith M: pVAC-Seq: A genome-guided in silico approach to identifying tumor neoantigens. Genome Med 8: 11, 2016.

16. Warren RL, Choe G, Freeman DJ, Castellarin M, Munro S, Moore R and Holt RA: Derivation of HLA types from shotgun sequence datasets. Genome Med 4: 95, 2012.

17. Ha G, Roth A, Khattra J, Ho J, Yap D, Prentice LM, Melnyk N, McPherson A, Bashashati A, Laks E, et al: TITAN: Inference of copy number architectures in clonal cell populations from tumor whole-genome sequence data. Genome Res 24: 1881-1893, 2014.

18. Rosenthal R, McGranahan N, Herrero J, Taylor BS and Swanton C: DeconstructSigs: Delineating mutational processes in single tumors distinguishes DNA repair deficiencies and patterns of carcinoma evolution. Genome Biol 17: 31, 2016.

19. Alexandrov LB, Nik-Zainal S, Wedge DC, Campbell PJ and Stratton MR: Deciphering signatures of mutational processes operative in human cancer. Cell Rep 3: 246-259, 2013.

20. Macintyre G, Goranova TE, De Silva D, Ennis D, Piskorz AM, Eldridge M, Sie D, Lewsley LA, Hanif A, Wilson C, et al Copy number signatures and mutational processes in ovarian carcinoma. Nat Genet 50: 1262-1270, 2018.

21. Middha S, Zhang L, Nafa K, Jayakumaran G, Wong D, Kim HR, Sadowska J, Berger MF, Delair DF, Shia J, et al: Reliable pan-cancer microsatellite instability assessment by using targeted next-generation sequencing data. JCO Precis Oncol 2017: PO.17.00084, 2017.

22. Sztupinszki Z, Diossy M, Krzystanek M, Reiniger L, Csabai I, Favero F, Birkbak NJ, Eklund AC, Syed A and Szallasi Z: Migrating the SNP array-based homologous recombination deficiency measures to next generation sequencing data of breast cancer. NPJ Breast Cancer 4: 16, 2018.
23. Chen EY, Tan CM, Kou Y, Duan Q, Wang Z, Meirelles GV, Clark NR and Ma'ayan A: Enrichr: Interactive and collaborative HTML5 gene list enrichment analysis tool. BMC Bioinformatics 14: 128, 2013

24. Lausen B, Hothorn T, Bretz F and Schumacher M: Assessment of optimal selected prognostic factors. Biom J 46: 364-374, 2004.

25. Hothorn T and Lausen B: On the exact distribution of maximally selected rank statistics. Comput Stat Data Anal 43: 121-137, 2003.

26. Takaya H, Nakai H, Takamatsu S, Mandai M and Matsumura N: Homologous recombination deficiency status-based classification of high-grade serous ovarian carcinoma. Sci Rep 10: 2757, 2020

27. Cancer Genome Atlas Network: Comprehensive molecular characterization of human colon and rectal cancer. Nature 487: 330-337, 2012

28. Zaidi SH, Harrison TA, Phipps AI, Steinfelder R, Trinh QM, Qu C, Banbury BL, Georgeson P, Grasso CS, Giannakis M, et al: Landscape of somatic single nucleotide variants and indels in colorectal cancer and impact on survival. Nat Commun 11: 3644 , 2020.

29. Franko J, Shi Q, Goldman CD, Pockaj BA, Nelson GD, Goldberg RM, Pitot HC, Grothey A, Alberts SR and Sargent DJ: Treatment of colorectal peritoneal carcinomatosis with systemic chemotherapy: A pooled analysis of north central cancer treatment group phase III trials N9741 and N9841. J Clin Oncol 30: 263-267, 2012.

30. Boeckx N, Koukakis R, Op de Beeck K, Rolfo C, Van Camp G, Siena S, Tabernero J, Douillard JY, André T and Peeters M Primary tumor sidedness has an impact on prognosis and treatment outcome in metastatic colorectal cancer: Results from two randomized first-line panitumumab studies. Ann Oncol 28: 1862-1868, 2017.

31. Ghiringhelli F, Hennequin A, Drouillard A, Lepage C, Faivre J and Bouvier AM: Epidemiology and prognosis of synchronous and metachronous colon cancer metastases: A French population-based study. Dig Liver Dis 46: 854-858, 2014.

32. Pennington KP, Walsh T, Harrell MI, Lee MK, Pennil CC, Rendi MH, Thornton A, Norquist BM, Casadei S, Nord AS, et al: Germline and somatic mutations in homologous recombination genes predict platinum response and survival in ovarian, fallopian tube, and peritoneal carcinomas. Clin Cancer Res 20: 764-775, 2014

33. Zhao EY, Shen Y, Pleasance E, Kasaian K, Leelakumari S, Jones M, Bose P, Ch'ng C, Reisle C, Eirew P, et al: Homologous recombination deficiency and platinum-based therapy outcomes in advanced breast cancer. Clin Cancer Res 23: 7521-7530, 2017.

34. Bylsma LC, Gillezeau C, Garawin TA, Kelsh MA, Fryzek JP, Sangaré L and Lowe KA: Prevalence of RAS and BRAF mutations in metastatic colorectal cancer patients by tumor sidedness: A systematic review and meta-analysis. Cancer Med 9: 1044-1057, 2020.

35. Wasserman I, Lee LH, Ogino S, Marco MR, Wu C, Chen X, Datta J, Sadot E, Szeglin B, Guillem JG, et al: SMAD4 loss in colorectal cancer patients correlates with recurrence, loss of immune infiltrate, and chemoresistance. Clin Cancer Res 25: 1948-1956, 2019.

36. Manzat Saplacan RM, Balacescu L, Gherman C, Chira RI, Craiu A, Mircea PA, Lisencu C and Balacescu O: The Role of PDGFs and PDGFRs in colorectal cancer. Mediators Inflamm 2017: 4708076, 2017.

This work is licensed under a Creative Commons Attribution-NonCommercial-NoDerivatives 4.0 International (CC BY-NC-ND 4.0) License. 